\title{
Diabetes und Syphilis.
}

\author{
Von \\ M. Simmonds. \\ (Aus dem Pathologischen Institut des Allgemeinen Kranken- \\ hauses St. Georg, Hamburg.)
}

Vor 10 Jahren berichtete ich im Ärztlichen Verein über 150 Sektionsfälle von Diabetes mellitus und kam dabei zum Schluß, daß in 90\% derselben ein pathologischer Befund am Pankreas festzustellen war. Die Veränderungen ließen sich 92 mal schon makroskopisch, 44 mal nur mikroskopisch nachweisen. Seitdem hat sich mein Material auf fast 300 Fälle vermehrt, ohne daß dabei das Gesamtresultat eine wesentliche Verschiebung erlitten hätte. Die angetroffenen Veränderungen waren wechselnd. Bei einer großen Anzahl von Individuen, besonders jüngeren, lag eine ätiologisch nicht erklärbare Atrophie des Organs vor, in einer zweiten Gruppe zeichnete sich das Pankreas durch einen starken Fettreichtum aus. In einer dritten Gruppe, zu der vorwiegend Individuen mit ausgebreiteter Arteriosklerose zählten, war eine mehr oder minder erhebliche Bindegewebsinduration mit Einengung der Drüsensubstanz festzustellen. In einer vierten kleineren Gruppe endlich hatte eine schwielige Verödung des Parenchyms von einer schweren chronischen Entzündung des Ductus Wirsungianus mit starker Konkrementbildung ihren Ausgang genommen.

Neben diesen schwieligen Atrophien, die sich im Anschluß an eine chronische Sialodochitis calculosa ausgebildet hatten, kamen nun in sehr spärlicher Zahl Fälle zur Beobachtung, in denen eine Teilnahme der Speichelgänge fehlte, und es blieb zunächst unklar, welcher Prozeß zur Verödung der Drüse geführt hatte.

Es handelte sich im ganzen um drei Fälle, über die ich kurz berichten will. Der erste (1916. 857) betraf einen 49 jährigen Diabetiker, der an Phthise gestorben war und in dessen Anamnese sich eine alte Lues vermerkt fand. Der zweite (1918. 850) betraf ebenfalls einen 40 Jahre alten Diabetiker, der syphilitisch infiziert gewesen war. Der dritte Patient (1919. 240) hatte ebenfalls neben Diabetes an Phthisis gelitten und war seinem Lungenleiden erlegen. In der Anamnese fehlten Angaben über Lues, doch ließ eine doppelseitige Fibrosis testis neben ausgesprochener glatter Atrophie des Zungengrundes bei dem 36jährigen Mann auf eine alte Syphilisinfektion schließen.

Die drei Präparate glichen einander so sehr, daß es unnötig ist, sie einzeln zu beschreiben. Die Bauchspeicheldrüse war jedesmal klein, 
hatte ein Gewicht von $26-36 \mathrm{~g}$, war fest mit der Umgebung verwachsen und stellte nur noch ein fibröses Gebilde dar, in der bis bohnengroße Drüsenreste erkennbar waren. Das Bindegewebe war im allgemeinen zellarm, nur an wenigen Stellen von Lymphocytenhaufen durchsetzt. Die kleinen Àrterien waren vielfach dickwandig, zeigten indes keinerlei für Syphilis spezifische Veränderungen.

Wenn auch somit die histologische Untersuchung keine Stütze für diese Annahme lieferte, so möchte ich doch im Hinblick auf das Fehlen aller anderen ätiologisch verwertbaren Momente den Schluß ziehen, daß in den drei Fällen die fibröse Atrophie des Organs auf eine abgelaufene syphilitische Pankreatitis mit Ausgang in Schrumpfung zurückzuführen war.

Was wissen wir bisher über syphilitische Erkrankungen der Bauchspeicheldrüse? Zunächst ist bekannt, daß eine syphilitische Pankreatitis nicht selten bei Säuglingen mit Lues congenita anzutreffen ist. Mitteilungen über Erkrankungen der Bauchspeicheldrüse bei erworbener Syphilis sind dagegen recht spärlich in der Literatur vorhanden. Ich kenne nur die von Thorel'), Schlagenhaufer ${ }^{2}$ ), $\mathrm{Koch}^{3}$ ), die in ihren Fällen neben chronisch entzündlichen Vorgängen auch Gummibildungen antrafen. Ich glaube indes nicht, daß man aus diesen spärlichen Angaben auf eine so überaus große Seltenheit der Pankreassyphilis schließen darf, da ich selbst zwei Präparate von Gummibildung der Bauchspeicheldrüse Erwachsener in meiner Sammlung besitze. Beide Male handelte es sich um Männer in den sechziger Jahren, von denen der eine an Tabes, der andere an Myocarditis gummosa gestorben. war. Bei dem einen war das ganze Organ derb, groß, sein Bindegewebe von Rundzellen reichlich durchsetzt, die Drüsensubstanz durch fibröse Züge auseinandergedrängt. Bei dem anderen fand sich ein haselnußgroßes Gummi im Pankreaskopfe. Trotz der schweren Veränderungen des Organs scheinen doch Zeichen einer Glykosurie gefehlt zu haben. Wenigstens ist in den Krankengeschichten, ebenso wie in den Fällen von Schlagenhaufer, Thorel und Koch nichts davon erwähnt. Allzuviel Gewicht möchte ich freilich auf die fehlende Angabe nicht legen. Für meine eingangs berichteten Fälle möchte ich jedenfalls den Diabetes mit der festgestellten syphilitischen Erkrankung des Pankreas in Verbindung bringen.

Die Frage nach einem Zusammenhang zwischen Diabetes und Syphilis ist oft behandelt, aber recht verschieden beantwortet worden. Während z. B. Oeser ${ }^{4}$ ) den Einfluß der Syphilis in der Ätiologie des Diabetes mellitus für zweifellos hält, und von einer chronischen Entzündung des Pankreas auf Grund luischer Infektion redet, spricht sich der auf diesem Gebiete sehr erfahrene Pathologe Herx hei mer ${ }^{5}$ ) äußerst vorsichtig aus. „Bei Luetikern“, sagt er, ,,ist öfter Diabetes festge- 
stellt worden. Ob hier aber ein Konnex besteht, speziell eine Pankreaserkrankung vorliegt, ist nicht sicher." Ich glaube, daß die Widersprüche sich ausgleichen, wenn man zugibt, daß ein Zusammenhang zwischen Syphilis und Diabetes wohl vorkommen kann, daß er aber im ganzen nur selten sich sicherstellen läßt.

Jedenfalls ist zuzugeben, daß das Zusammentreffen von Diabetes und Syphilis kein häufiges ist. Unter meinen 300 Fällen von Diabetes, die ich am Sektionstisch beobachten konnte, war nur in $6 \frac{1}{2} \%$ Syphilis mit Sicherheit nach Anamnese oder anatomischem Befund festzustellen. Das würde im Hinblick auf die Häufigkeit der Luesbefunde bei unseren Autopsien den Schluß zulassen, daß die Syphilis in der Ätiologie des Diabetes keine wesentliche Rolle spielt. Wir dürfen weiter nur dann einen Zusammenhang zwischen Zuckerharnruhr und vorausgegangener Lues annehmen, wenn tatsächlich im Pankreas Veränderungen angetroffen werden, die mit Wahrscheinlichkeit als Folgen der Syphilis anzusprechen sind.

Unter den 20 Fällen meiner Zusammenstellung, in denen Lues und Diabetes gleichzeitig festgestellt worden war, befanden sich nur 17, in denen ein Zusammenhang nicht nachweisbar waren. 4 mal handelte es sich dabei um eine schwere Adipositas des Organs, $8 \mathrm{mal}$ um eine einfache Atrophie ohne Residuen eines entzündlichen Prozesses, 5 mal um eine diffuse Induration, die auf die begleitende Arteriosklerose bezogen werden durfte. Es lag also in 17 Fällen kein Anlaß vor, die angetroffene Pankreasveränderung mit der konstatierten Lues in Zusammenhang zu bringen. Diabetes und Syphilis trafen in diesen 17 Fällen nur zufällig zusammen.

Anders liegen die Verhältnisse in den 3 anfangs geschilderten Fällen Hier fanden sich ausgedehnte Residuen einer schweren chronischen Entzündung des Pankreas vor, für die weder in der Drüse selbst noch in der Umgebung ein Ausgang zu finden war. Im Hinblick auf die sichergestellte frühere Luesinfektion zögerte ich daher nicht, die Pankreasveränderung als eine spezifische aufzufassen. In diesen 3 Fällen war also ein Zusammenhang zwischen Diabetes und Syphilis anzunehmen. Bedenkt man aber, daß es sich um 3 von 300 Fällen, also nur um $1 \%$ derselben handelte, so muß man zugeben, daß die Syphilis in der Ätiologie des Diabetes eine sehr untergeordnete Rolle spielt.

\section{Literatur.}

1) Thorel, Virchows Archiv. 158, 271. - $\left.{ }^{2}\right)$ Schlagenhaufer, Dieses Archiv. 31, 43. u 59, 377. - 3) Koch, Virchows Archiv 214, 180. - 4) Oeser, Nothnagel Handb. 18 II. S. 121. - ${ }^{5}$ ) Her xhe i mer, Lubarsch-Ostertag Jahresber. 11 I, S. 286. 